\title{
Conversion of light into macroscopic helical motion
}

Supitchaya Iamsaard ${ }^{1}$, Sarah J. A $\beta$ hoff ${ }^{1}$, Benjamin Matt ${ }^{1}$, Tibor Kudernac, ${ }^{2}$ Jeroen J. L. M. Cornelissen $^{1}$, Stephen P. Fletcher ${ }^{3}$ and Nathalie Katsonis ${ }^{1 *}$

1 Laboratory for Biomolecular Nanotechnology, MESA+ Institute for Nanotechnology, University of Twente, PO Box 207, 7500 AE Enschede, The Netherlands.

${ }^{2}$ Laboratory for Molecular Nanofabrication, MESA+ Institute for Nanotechnology, University of Twente, PO Box 207, 7500 AE Enschede, The Netherlands.

${ }^{3}$ Department of Chemistry, Chemistry Research Laboratory, University of Oxford, 12 Mansfield Road, Oxford OX1 3TA, UK.

*Correspondence to: n.h.katsonis@utwente.nl; stephen.fletcher@chem.ox.ac.uk

A key goal of nanotechnology is the development of artificial machines capable of converting molecular movement into macroscopic work. While conversion of light into shape changes has been reported and compared to artificial muscles, real applications require work against an external load. Here, we describe the design, synthesis and operation of spring-like materials capable of converting light energy into mechanical work at the macroscopic scale. These versatile materials consist of molecular switches embedded in liquid-crystalline polymer springs. In these springs, molecular movement is converted and amplified into controlled and reversible twisting motion. The springs display complex motion including winding, unwinding and helix inversion as dictated by their initial shape. Importantly, they can produce work by moving a macroscopic object and mimicking mechanical movements, such as those used by plant tendrils to help the plant access sunlight. These functional materials have potential applications in micro-mechanical systems, soft robotics and artificial muscles.

The promise of developing artificial molecular machines has inspired scientists for decades ${ }^{1,2}$ and led to the design and operation of small molecules that exhibit mechanically relevant movements such as rotation, ${ }^{3-6}$ walking $^{7}$ and twisting, ${ }^{8}$ as well as more complex molecular-scale operations such as driving ${ }^{9}$ and chemical synthesis. ${ }^{10}$ Rudimentary examples of nanomachines capable of work $^{11-15}$ provide tantalizing hints of the potential power of molecular devices. However, materials capable of translating molecular motion into useful macroscopic function remain elusive. ${ }^{16-19}$

Nature uses molecular-scale machines to drive every significant biological process ${ }^{20}$ and powers macroscopic mechanical motion in plants in highly complex processes. ${ }^{21}$ Examples of biological systems built on helical motion include powerful engines such as spasmoneme springs, ${ }^{21}$ seed pod opening ${ }^{22}$ and tendril coiling. ${ }^{23}$ 
Based on the general concept that such plant-like helical deformations may similarly occur in artificial systems, thus making them capable of producing mechanical work, we set out to design a system where molecular-movement would be translated to rotational movement across scalelengths, and form the basis of macroscopically functional materials. Previous studies have reported on a light-induced film twisting of thin crystals ${ }^{24,25}$ and out-of-plane deformations of polymer films controlled by irradiation with polarized light ${ }^{26}$. In these examples, selective irradiation was necessary to control the twisting mechanism. In contrast, here we seek to create smart materials whose photo-response is inherently encoded in their structure.

\section{Results and Discussion}

Amplifying molecular chirality in a liquid crystal polymer network. Our approach involves using a liquid crystal polymer network ${ }^{27}$ that can selectively form either right-handed or lefthanded macroscopic helices at room temperature. Liquid crystal polymer networks display a strong coupling between orientational order and mechanical strain, which is why they undergo deformations when submitted to a stimulus-induced decrease of order. Light is an especially attractive stimulus to induce such macroscopic modifications, as it has proven to be a versatile and controllable energy source for driving a wide range of nanoscale molecular systems. ${ }^{11,12,15-18}$ Arguably, the most straightforward manner to induce light-responsiveness to a polymer network is to introduce light-responsive switches, such as azobenzene units, ${ }^{28-30}$ into its covalent structure. Molecular switch 1 (Fig. 1a) is an azobenzene-based photochromic switch that undergoes trans-cis photo-isomerization under irradiation with UV light (Fig. S1). The switch acts as a nanoscale energy converter and is incorporated into the polymer network through polymerizable acrylate groups. It has been shown that for azobenzene concentrations up to 10 $\mathrm{wt} \%$, photoinduced deformations scale with the concentration of the azobenzene ${ }^{31}$, thus the concentration of $\mathbf{1}$ in the polymer network was set to $10 \mathrm{wt} \%$ to provide large photo-induced deformations.

The host matrix we used is based on a combination of a low molecular-weight nematic liquid crystal (commercially available E7) and acrylate-functionalized nematic liquid crystals that photo-polymerize in the presence of an initiator (Fig. S2). We selected a photo-initiator that absorbs at wavelengths longer than $420 \mathrm{~nm}$ to achieve cross-polymerization without isomerization of 1. A small amount of the chiral dopant (S-811, Fig. 1b) was added to the liquid crystalline mixture to favor a left-handed twist (Fig. 1c). The mixture is then introduced into a glass cell promoting a twist geometry, in which the orientation of the liquid crystal director changes smoothly by $90^{\circ}$ from the bottom surface to the top surface (Fig. 1c,d). Polarized optical microscopy shows that the molecular organization of the liquid crystal is largely preserved after polymerization. After the cells were opened and the films dried, strips with a width of 0.7-0.9 $\mathrm{mm}$ were cut in a direction characterized by the angular offset $\varphi$ defined here as the angle between the orientation of the molecules at mid-plane on one hand, and the cutting direction on the other hand, which corresponds to the long axis of the ribbons also (Fig. 2). An illustration of how the films are cast and how they coil spontaneously into springs once they are cut into ribbons is provided in Figure S3. Below we show that the direction in which the ribbons are cut is a parameter determining not only the pitch and handedness of the helical shapes that are formed, but also their photo-responsive behavior. 
Generating a diversity of helical shapes. Upon polymerization and/or drying of such a film, anisotropic shrinking occurs and leads to out-of-plane twisting once the film is released from the cell and cut into ribbons ${ }^{32,33,34}$. In particular, Urayama and coworkers have presented a strain model according to which ribbons cut out from twisted nematics select a helicoid or spiral shape depending on their width. ${ }^{32}$ In other words, polymerization and/or drying of the ribbons induce shrinking of each face of the ribbon orthogonally to each other, and the ribbons have to curl in order to accommodate for it. Because the shrinking is anisotropic, the shape of each ribbon is determined by the orientation of the molecules with respect to the short and long axes of the ribbons.

In the work reported here, the width of the ribbons is constant. We propose that the diversity of helical shapes that we observe arises from three sources, which can add-up or compete depending on the angle at which the ribbon is cut: (i) the handedness of the director twist, which is determined by the handedness of the chiral dopant; (ii) the angular offset $\varphi$, which is determined by the angle at which the ribbon is cut; and (iii) moreover, a gradient in density is expected through the thickness of the sample, because the films are always irradiated from the same side, i.e. from the top of the sample, during photo-polymerization. Based on the competition between these sources of asymmetry, the samples can form a rich variety of chiral shapes, from right-handed or left-handed ribbons with diverse pitches (Fig. 2, ribbon B and D), to ribbons for which chirality is not expressed macroscopically (Fig. 2, flat ribbon A or open ring ribbon $\mathrm{C}$ ). Additional images for the shape selection of the ribbons are shown in Supplementary Figure S4.

As expected, in ribbons where the chiral dopant S-811 is replaced by its enantiomer R-811 we observe that the shape of the ribbons is the mirror image - that is the polymers produced are enantiomers of each other (Fig. S5). For example, an S-811 ribbon cut at $\varphi=22^{\circ}$ is expected to be the enantiomer of an R-811 ribbon cut at $\varphi=158^{\circ}$ (Fig. 3), as both the handedness of the director twist and the angular offset are symmetric in these two ribbons. Indeed, we observe that the former ribbon yields a left-handed spring, whereas the latter yields a right-handed spring having a similar pitch (Fig. S4,S5).

In passive (non-photoactive) ribbons that have been emptied from chiral molecules and nonreacted materials, twist-free ribbons (either open rings or flat ribbons), have been observed at $\varphi \approx$ $45^{\circ}$ and $135^{\circ}$, which is expected for a non-chiral system of orthogonally shrinking sheets ${ }^{35}$. In contrast, we observed achiral ribbons for $\varphi \approx 0^{\circ}$ and $90^{\circ}$, where the surface directors are not parallel to the axes of the ribbon but instead, the mid-plane director is parallel to one of the axes. This difference indicates a strong source of asymmetry in the system. To verify this conclusion, we heated up the ribbons up to $180{ }^{\circ} \mathrm{C}$ (Fig. S6,S7). As the temperature is gradually increased, unreacted materials (E7 and chiral dopant) are expelled from the polymer network. When all these materials have diffused away, the ribbons are emptied from the additional source of asymmetry provided by S-811 and undergo a shape transition. At $180^{\circ} \mathrm{C}$, the shapes we observe correspond well to the shapes of the passive ribbons observed by Sawa et al.; in particular, the shape of the $\varphi=45^{\circ}$ ribbon becomes achiral. ${ }^{32,33}$ Importantly, the temperature-induced shape transition was not reversible.

Surprisingly, upon cutting all the ribbons presented here curl in such a way that the area facing the top of the cell is always found on the outside of the ribbon, whereas the bottom of the film always ends up inside the twisted ribbon. This conclusion was drawn after labeling one side of the ribbons with sticky tape (Fig. S8,S9). We propose that the preferential curling direction 
originates in a light intensity gradient due to the thickness of the sample during photopolymerization, because both the photoactive dopant and the photo-initiator are optical absorbers at $\lambda \geq 420 \mathrm{~nm}$ (Fig. S1). As the polymerization rate scales with light intensity, the reactive monomers are depleted preferentially at the top of the sample. This gradient of concentration in reactive monomers drives the diffusion of the monomers to the top of the film during polymerization and the final sample displays a higher density and cross-link density at the top. ${ }^{36}$ In order to confirm that the curvature of the ribbons is influenced by a density gradient in the film, we performed photo-polymerization by placing the lamp at the bottom of the sample rather than at its top. For a fixed offset angle, we observed that the ribbons had a similar pitch, but an opposite handedness. We find that when photo-polymerization occurs from the bottom, it is the bottom of the film that always ended up outside the spiral ribbons. This observation provides insight into both the shape and the photo-responsive properties of the springs. In all experiments described below, the ribbons were prepared by photo-polymerization from the top of the cell.

To summarize, two parameters dictate shape selection in the photo-responsive ribbons - the pitch and the bending direction. The pitch of the spiral ribbons (i.e. the absolute value of the curvature) is determined by the director orientation within the ribbons, via anisotropic shrinkage. The bending direction, i.e. the handedness of the spiral ribbons, is determined not only by the director orientation within the ribbons, but also by the direction of the polymer gradient.

Photo-actuation modes of the helical ribbons. We investigated the photo-response of these twisted ribbons (Fig. 4). Under irradiation with light, left-handed spiral ribbons doped with S811 decrease in their macroscopic pitch, while the corresponding right-handed ribbons show an increase in macroscopic pitch (Fig. S4, Supplementary Movies 1\&2). Remarkably, it was also possible to observe inversion of helical sense from right-handed to left-handed (Fig. 4a). After a few minutes the deformations reach a steady state $\left(\lambda=365 \mathrm{~nm}\right.$ at $\left.60 \mathrm{~mW} / \mathrm{cm}^{2}\right)$. They are fully reversible at room temperature, with relaxation times of the order of a dozen minutes. This reversal process can be accelerated to completion in a few seconds with visible light. The winding of the photo-responsive ribbons was associated with remarkably large isochoric contraction (reaching up to $50 \%$ of the original length of the ribbons). For unwinding associated with elongation, amplitudes of up to $40 \%$ of the original shape were observed (Fig. $4 \mathrm{~b}$ ). The direction of the actuation (expansion or contraction) and its magnitude are both encoded in the shape and geometry of each spiral ribbon.

Important characteristics of an actuator are rate of response and reversibility. In the case of photo-isomerization reactions, the intensity of irradiation constitutes a control parameter for reaction rate. On the other hand, it is likely that the rate of the shape reversal is determined by the rate of thermal relaxation of the photoactive dopant, the cis-azobenzene molecule. Here, the half-life time for thermal relaxation of the cis-azobenzene is about $15 \mathrm{~min}$ in solution and in ambient conditions (Fig. S1). This half-life corresponds well to the thermal relaxation rate of the ribbons, which suggests that the reorganization of the polymer network is faster than the thermal isomerization of the cis-azobenzene. The relaxation process is thus likely determined by the specific molecular structure of the photo-responsive switch. The thermal relaxation can be accelerated by irradiation with visible light. We also performed absorption spectroscopy of the films upon repeated alternating irradiation with UV $(\lambda=365 \mathrm{~nm})$ and visible $(\lambda \geq 420 \mathrm{~nm})$ light. Photo-switching of azobenzene $\mathbf{1}$ is indeed reversible, even after being covalently bonded to the 
polymer network, and there is nearly no fatigue effect on the sample after ten cycles of alternating UV and visible irradiation (Fig. S10).

The mechanical behavior of the springs depends on the handedness of the director twist and on their angular offset $\varphi$, (Fig. 3). Earlier studies demonstrated that controlling the geometry of the director and its spatial variation in photo-responsive polymer networks can drive various types of light-induced deformations in these materials ${ }^{26,30}$. Here we show that large deformations are possible by utilizing the twisting mechanism. Understanding this mechanism requires taking into account the specificity of light as a stimulus. Both experimental and theoretical investigations have established that irradiating liquid crystal polymer networks doped with photo-responsive molecules induces disorder and thus leads to anisotropic deformations characterized by contraction in the direction of the director, and expansion occurring in perpendicular directions (Fig. 4c). ${ }^{37}$ As mentioned above, all ribbons curl in such a way that the region that was facing the top of the cell during polymerization is always found at the outside of the ribbon, whereas the bottom of the film always ends up inside the twisted ribbon. Having determined the orientation of the molecules on each side of the ribbon before irradiation, an interpretation becomes possible: the ribbons always deform to accommodate the preferred distortion along the main axis of the ribbon, and this preferred distortion is determined by the orientation of the molecules, which is in turn determined by the cutting direction.

The mechanism driving photo-response in the ribbons is illustrated for the ribbon where $\varphi=45^{\circ}$ (Fig. 4d). Here, the outside face undergoes expansion along its long axis, whereas the inner face shrinks. Both photo-deformations accommodate an increase in the twist, as observed experimentally (Fig. 4a). The work provided by such a ribbon can be estimated by attaching a known mass to one of the springs. Under irradiation, the ribbon works against gravity by lifting the mass up (Fig. S11). This experiment yielded a value of $3.04 \mathrm{~J} / \mathrm{mol}$ of azobenzene (and a power of $9 \mathrm{nW})$, a similar value to that reported for the work by thin molecular crystals (2.95 $\mathrm{J} / \mathrm{mol}$ of photo-responsive molecule embedded in the crystal). ${ }^{15}$ The similarity between these values highlights that these soft actuators can perform at least as well as their rigid counterparts. To summarize, the photo-response of the ribbons at the macroscopic level is determined by the orientation of the molecules within the ribbons. Under irradiation with UV light, the ribbons contract along the director and expand in the perpendicular directions, as is consistent with an $\mathrm{UV}$-induced increase of disorder. Notably, this mechanism is reminiscent of a design strategy implemented successfully in other photomechanical soft materials: In Aida et al.'s polymer brushes, irradiation with light unbalances competing strains between the front and back sides of the film, which in turn bends the film. ${ }^{38}$

Toward mimicking the mechanical behavior of plant tendrils. In the movements of plant tendrils, winding and unwinding is understood to be governed by the shrinking and expansion of cell walls orthogonally to each other. In our system, we anticipate that the mechanism of winding and unwinding is due to photo-isomerization of embedded units of $\mathbf{1}$ from the trans-state, to the bent cis-state - contracting the material along, and elongating perpendicular to - the director. This proposed mechanism bears considerable resemblance to that believed to occur in biological systems.

In nature, tendrils combine bending and twisting distortion modes to find a support and lift a plant upwards, towards the Sun. A fully operational device cut out of a single sheet of liquid crystal polymer network, was found to display the type of complex and biologically-relevant behavior that inspired the current approach (Fig. 5). These mixed-helicity springs, comprising 
two oppositely handed helices joined at a kink, display regions that are simultaneously coiling and uncoiling under irradiation (Fig. 5b,c). Noticeably, when a hybrid spring was clamped at both ends and pulled axially, it unwound. Furthermore, localized irradiation of specific sections of these springs leads to a bending motion whose direction can be controlled also (Fig. 5d).

Finally, the differential behavior of a mixed-helicity spring was used to demonstrate proof-ofprinciple for a photo-mechanical engine that performs a complex mechanical function. To this end, a tendril-like spring was clamped at each end and irradiated alternately with UV and visible light (Fig. 6). The confinement of the tendril allows the central kink to perform a continuous push-pull motion that is similar to the piston motion which is essential to the design and functioning of classical mechanical motors. The movement of the spring retained its amplitude over 10 cycles (Movie 3). As a further demonstration of the ability of these materials to produce useful work we attached a simple magnet to the central kink of a tendril-like spring. The material was found to withstand the weight of the magnet ( $\mathrm{m} \approx 2 \mathrm{mg}$ ) with only a small reduction in mobility, and the movement of this magnet was used to move an additional magnet $(\mathrm{m} \approx 0.5$ $\mathrm{mg}$ ), separated (by $\sim 10 \mathrm{~mm}$ ) through a glass plate, back and forth in a predictable way that was completely controlled by the mechanical action of the mixed-helicity spring. (Fig. 6c and Movie 4).

Conclusion. Inspired by biological systems, we have designed, synthesized and studied the versatile actuation modes of photo-responsive liquid crystalline polymer springs. Light operates molecular scale motion (cis-trans photo-isomerization) which is converted into large macroscopic deformations of the springs, through a mechanism which involves an increase of disorder associated to anisotropic deformations. This molecular approach gives access to (and control of) a large diversity of morphology and photo-responses in the spiral ribbons. More than a single actuation mode is inherently encoded in these chiral objects and the actuation can be reversed when changing from one handedness to the other. Moreover, we have demonstrated that these chiral objects can be used to achieve work. These versatile actuators constitute a solid basis for designing novel functional materials that can provide an economic, clean and electrode-less alternative to conventional actuators.

\section{Methods}

Materials. Monomers 2, 3 and $\mathbf{4}$ were purchased from Synthon Chemicals and mixed in a 3:1:2 ratio by weight. A nematic liquid crystal (E7 Merck), used to prevent crystallization when the mixture was inserted in the cell, was added to the monomer mixture in a ratio of 1:2.5 by weight in favor of the monomer mixture. Molecular switch 1 was synthesized according to reported procedures ${ }^{39,40}$ and its photo-isomerization in hexane was characterized using a Perkin Elmer Lambda 850. 1 was added in 10 $\mathrm{wt} \%$ to the matrix previously described. Chiral dopant S-811 or R-811 (Merck) was added to the mixture $(0.04 \mathrm{wt} \%)$ to induce a cholesteric helix with a pitch of $200 \mu \mathrm{m}$. Phenylbis(2,4,6trimethylbenzoyl)phosphine oxide (Ciba Irgacure 819) was purchased from Sigma-Aldrich and used as a photoinitiator with a $1.0 \mathrm{wt} \%$ concentration in the cholesteric mixture. All compounds were dissolved and mixed in dichloromethane purchased from Sigma-Aldrich. Afterwards the solvent was evaporated at $40^{\circ} \mathrm{C}$ under a nitrogen stream. Twist cells with a $50 \mu \mathrm{m}$ cell gap were either purchased from EHC or homemade using a Sunever 150 alignment layer from Nissan Chemical Industries. The twist cells were preheated at 
$55^{\circ} \mathrm{C}$ prior to their use and the cholesteric mixture was introduced into the cell at $48^{\circ} \mathrm{C}$. The cell was irradiated from the top using an Edmund MI-150 High-intensity Illuminator equipped with a cut-off filter $(\lambda \geq 420 \mathrm{~nm})$ to initiate the polymerization. After irradiation the cell was left in the oven at $55^{\circ} \mathrm{C}$ overnight. An illustration for this procedure is provided Figure S3. Thermal gravimetric analysis (TGA) experiments show that the final ribbon contains $\sim 25 \%$ E7 in mass, suggesting that all the material composing the photo-responsive liquid crystalline mixture is still present in the final ribbons (Fig. S12).

Preparation of the ribbons and structural characterization. The twist cells were frozen with liquid nitrogen and then opened using a scalpel to reveal the polymer film. The film was cut in different directions to provide ribbons with a variety of shapes. After cutting, the ribbons were dried at $55^{\circ} \mathrm{C}$ for 2 $\mathrm{h}$. The width of the ribbons was adjusted to $\sim 0.8 \mathrm{~mm}$. The length of the one-handed ribbons was $\mathrm{L} \sim 0.8-$ $1.3 \mathrm{~cm}$ and the width of the mixed-helicity ribbons was $\mathrm{L} \sim 4 \mathrm{~cm}$. The thickness of the ribbons and the molecular organization through the film were evaluated by using scanning electron microscopy (SEM). The thicknesses were $43 \mu \mathrm{m}$ for films prepared in commercial cells and $48 \mu \mathrm{m}$ for those prepared in homemade cells, in good agreement with the $50 \mu \mathrm{m}$ gap of the cells that were used. A Hönle blue point LED lamp was used to analyze the photo responses. Therefore the polymer ribbon was placed $4 \mathrm{~cm}$ below one LED head and irradiated with an intensity ranging from $30 \mathrm{~mW} / \mathrm{cm}^{2}$ to $60 \mathrm{~mW} / \mathrm{cm}^{2}$. The light-driven deformations were followed using a Dino-Lite Pro AM4113T USB microscope with 29x magnification.

\section{References}

1. Feynman, R. P. in Miniaturization, H. D. Gilbert, Ed. (Reinhold, New York, 1961).

2. Drexler, K. E. Nanosystems: Molecular Machinery, Manufacturing and Computation (Wiley, New York, 1992).

3. Koumura, N., Zijlstra, R. W. J., van Delden, R. A., Harada, N. \& Feringa, B. L. Light-driven monodirectional molecular rotor. Nature 401, 152-155 (1999).

4. Leigh, D. A., Wong, J. K. Y., Dehez, F. \& Zerbetto, F. Unidirectional rotation in a mechanically interlocked molecular rotor. Nature 424, 174-179 (2003).

5. Fletcher, S. P., Dumur, F., Pollard, M. M. \& Feringa, B. L. A reversible, unidirectional molecular rotary motor driven by chemical energy. Science 310, 80-82 (2005).

6. Balzani, V. et al. Autonomous artificial nanomotor powered by sunlight. Proc. Natl. Acad. Sci. USA 103, 1178-1183 (2006).

7. von Delius, M., Geertsema, E. M. \& Leigh, D. A. A synthetic small molecule that can walk down a track. Nature Chem. 2, 96-101 (2010).

8. Muraoka, T., Kinbara, K. \& Aida, T. Mechanical twisting of a guest by a photoresponsive host. Nature 440, 512-515 (2006).

9. Kudernac, T. et al. Electrically driven directional motion of a four-wheeled molecule on a metal surface. Nature 479, 208-211 (2011).

10. Lewandowski, B. et al. Sequence-specific peptide synthesis by an artificial small-molecule machine. Science 339, 189-193 (2013).

11. Berná, J. et al. Macroscopic transport by synthetic molecular machines. Nature Mater. 4, 704-710 (2005). 
12. Eelkema, R. et al. Nanomotor rotates microscale objects. Nature 440, 163 (2006).

13. Yamada, M. et al. Photomobile polymer materials: towards light-driven plastic motors. Angew. Chem. Int. Ed. 47, 4986-4988 (2008).

14. Juluri, B. K. et al. A mechanical actuator driven electrochemically by artificial molecular muscles. ACS Nano 3, 291-300 (2009).

15. Morimoto, M. \& Irie, M. A diarylethene cocrystal that converts light into mechanical work. J. Am. Chem. Soc. 132, 14172-14178 (2010).

16. Browne, W. R. \& Feringa, B. L. Making molecular machines work. Nat. Nanotechnol. 1, 2535 (2006).

17. Ariga, K., Mori, T. \& Hill, J. P. Mechanical control of nanomaterials and nanosystems. $A d v$. Mater. 24, 158-176 (2012).

18. Coskun, A. et al. Great expectations: can artificial molecular machines deliver on their promise? Chem. Soc. Rev. 41, 19-30 (2012).

19. Spinks, G. M. Deforming materials with light: photoresponsive materials muscle in on the action. Angew. Chem. Int. Ed. 51, 2285 - 2287 (2012).

20. Schliwa, M. Ed. Molecular Motors (Wiley, Weinheim, Germany, 2003).

21. Mahadevan, L \& Matsudaira, P. Motility powered by supramolecular springs and ratchets. Science 288, 95-99 (2000).

22. Armon, S., Efrati, E., Kupferman, R. \& Sharon, E. Geometry and mechanics in the opening of chiral seed pods. Science 333, 1726-1730 (2011).

23. Gerbode, S. J., Puzey, J. R., McCormick, A. G. \& Mahadevan, L. How the cucumber tendril coils and overwinds. Science 337, 1087-1091 (2012).

24. Zhu, L., Al-Kaysi, R. O. \& Bardeen, C. J. Reversible photoinduced twisting of molecular crystal microribbons. J. Am. Chem. Soc. 133, 12569-12575 (2011).

25. Kitagawa, D., Nishi, H. \& Kobatake, S. Photoinduced twisting of a photochromic diarylethene crystal, Angew. Chem. Int. Ed. 52, 9320-9322 (2013).

26. Lee, K. M. et al. Photodriven, flexural-torsional oscillation of glassy azobenzene liquid crystal polymer networks. Adv. Funct. Mater. 21, 2913-2918 (2011).

27. Warner, M. \& Terentjev, E. M. Liquid Crystal Elastomers (Oxford University Press, Oxford, 2003)

28. Natansohn, A. \& Rochon, P. Photoinduced motions in azo-containing polymers. Chem. Rev. 102, 4139-4175 (2002).

29. Yu, Y., Nakano, M. \& Ikeda, T. Directed bending of a polymer film by light. Nature $\mathbf{4 2 5}$, 145 (2003).

30. Van Oosten, C. L., Bastiaansen, C. W. M. \& Broer, D. J. Printed artificial cilia from liquidcrystal network actuators modularly driven by light. Nature Mater. 8, 677-682 (2009). 
31. Van Oosten, C. L., Harris, K. D., Bastiaansen, C. W. M. \& Broer, D. J. Glassy photomechanical liquid-crystal network actuators for microscale devices. Eur. Phys. J. E 23, 329-336 (2007).

32. Sawa, Y. et al. Shape selection of twist-nematic-elastomer ribbons. Proc. Natl. Acad. Sci. USA 108, 6364-6368 (2011).

33. Sawa, Y. et al. Shape and chirality transitions in off-axis twist nematic elastomer ribbons, Phys. Rev. E 88, 022502 (2013).

34. Teresi, L. \& Varano, V. Modeling helicoid to spiral-ribbon transitions of twist-nematic elastomers. Soft Mater 9, 3081-3088 (2013).

35. Forterre, Y. \& Dumais, J. Generating helices in nature. Science 333, 1715-1716 (2011).

36. van Oosten, C. L. et al. Bending dynamics and directionality reversal in liquid crystal network photoactuators. Macromolecules 41, 8592-8596 (2008).

37. Harris, K. D. et al. Large amplitude light-induced motion in high elastic modulus polymer actuators. J. Mater. Chem. 15, 5043-5048 (2005).

38. Hosono, N. et al., Large-area three-dimensional molecular ordering of a polymer brush by one-step processing. Science 330, 808-811 (2010).

39. Mossety-Leszczak, B., Wlodarska, M., Galina, H. \& Bak, G. W. Comparing liquid crystalline properties of two epoxy compounds based on the same azoxy group. Mol. Cryst. Liq. Cryst. 490, 52-66 (2008).

40. Li, C. et al. Synthesis of a Photoresponsive liquid-crystalline polymer containing azobenzene. Macromol. Rapid Commun. 30, 1928-1935 (2009).

\section{Acknowledgments}

This work was financially supported by the European Research Council (Starting Grant 307784 to N.K), the Netherlands Organisation for Scientific Research (a Vidi grant to N.K), and The Royal Society UK (an International Exchanges Grant to S.P.F \& N.K).

\section{Author Contributions}

N.K. and S.P.F. conceived the research. N.K., T.K. and J.C. guided the research. S.I. and B.M synthesized 1. S.I., S.J.A. and B.M. carried out the experiments. All authors discussed the results and commented on the manuscript at all stages.

\section{Figure Legends}

Figure 1. A photo-responsive liquid crystal in a twist-nematic molecular organization. a, The molecular photo-switch $\mathbf{1}$ used in this study is an azobenzene derivative. b. Chiral dopants S-811 and R-811 inducing a left-handed and right-handed twist in the liquid crystal, respectively. c, Molecular organization in the twist nematic cell [top view] and the angular offset $\varphi$, which characterizes the angle at which the ribbon is cut. The orientation of the molecules at mid-plane is shown with a double headed arrow. The cutting direction, which is also the long axis of the 
ribbon, is represented by a dotted line. The elongated rods represent molecules. d, The twist nematic molecular orientation through the thickness of the film [side view].

Figure 2. The ribbons display a variety of shapes depending on the direction in which they are cut. The samples display a rich variety of chiral shapes, from right-handed or left-handed ribbons (ribbon B and D), to ribbons for which chirality is not expressed macroscopically (flat ribbon $\mathrm{A}$ or open ring $\mathrm{C}$ ).

Figure 3. Shape and photo-actuation modes of the polymer springs as a function of the angular offset. The angular offset $\varphi$ is defined here as the angle between the orientation of the molecules at mid-plane and the cutting direction. The experimental error is estimated to $\pm 2^{\circ}$. $\mathbf{a}$, Ribbons doped with S-811, where the director twist is left-handed. b, Ribbons doped with R-811, where the director twist is right-handed. The two graphs are symmetric.

Figure 4. Photo-actuation modes of the polymer springs doped with S-811. a, Spiral ribbons irradiated 2 min with UV light $(\lambda=365 \mathrm{~nm})$ display isochoric winding, unwinding and helix inversion as dictated by their initial shape and geometry. $\mathbf{b}$, A large amplitude elongation is obtained upon light-driven unwinding of ribbons with $\varphi \sim 45^{\circ}$, and a large amplitude contraction is obtained upon light-driven unwinding of ribbons with $\varphi \sim 90^{\circ}$ ). c, Under irradiation with UV light, the ribbons contract along the director and expand in the perpendicular directions, as is consistent with an UV-induced increase of disorder. d, Scheme representing the mechanisms through which the shape of the ribbons is modified under irradiation with UV light, for $\varphi=45^{\circ}$. The ribbons deform to accommodate the preferred distortion along the main axis of the ribbon, and this preferred distortion is determined by the orientation of the molecules.

Figure 5. Mixed-helicity springs doped with S-811 display a complex range of mechanical photo-responses. a, A coiled tendril of the wild cucumber plant. b, The liquid crystal polymer film is cut to introduce regions that display different dynamic behavior. c, A polymer spring displaying a cucumber tendril-like shape, comprised of two oppositely handed helices connected by a kink. Upon irradiation the right-handed helix unwinds and $\mathbf{d}$, the left-handed helix winds. e, Bending of the spring is achieved and controlled by selective irradiation, that induces a local elongation of the right-handed ribbon. The colored circles represent the irradiation spots, where elongation occurs.

Figure 6. Proof-of-principle for a mechanical device powered by light. a, Upon alternate irradiation with UV and visible light, the central kink of a mixed-helicity ribbon doped with S811 performs a continuous piston-like motion (series of video frames). $\mathbf{b}$, The device displays no sign of fatigue over ten cycles of alternating irradiation. $\mathbf{c}$, A magnet connected to the kink $(\mathrm{m} \approx$ $2 \mathrm{mg}$ ) undergoes push-pull shuttling motion, the motion is further transmitted to another magnet placed $10 \mathrm{~mm}$ below $(\mathrm{m} \approx 0.5 \mathrm{mg}$ ). 
Figure 1

a

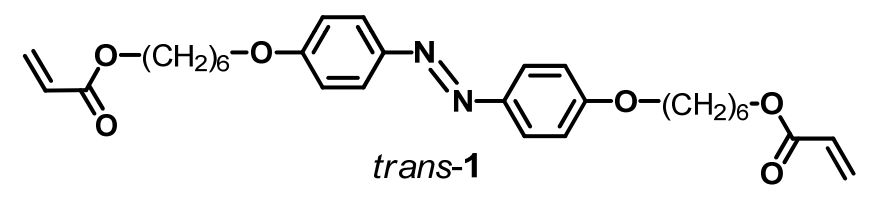

4f $\mathrm{h} v=365 \mathrm{~nm}$

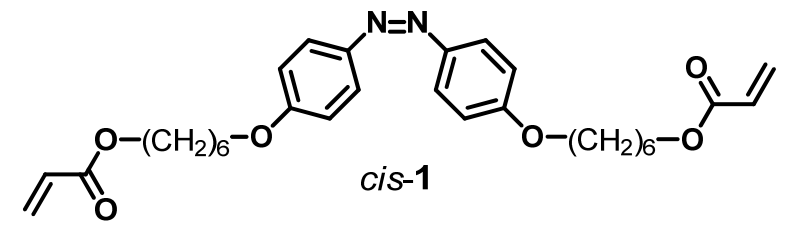

C

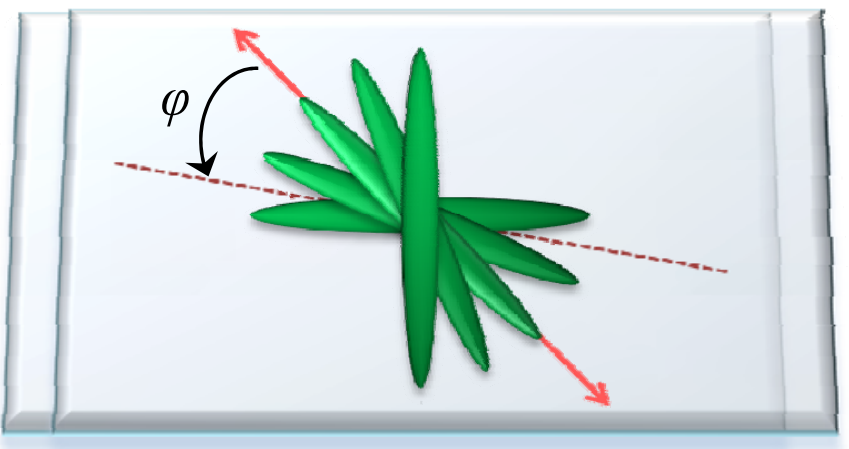

b

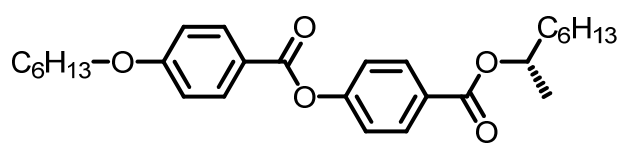

S-811

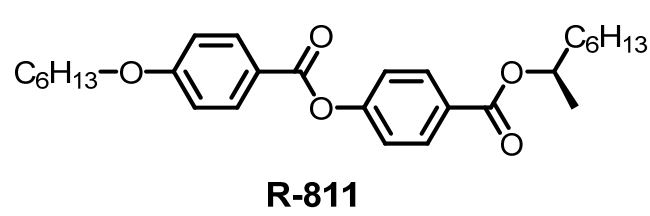

d

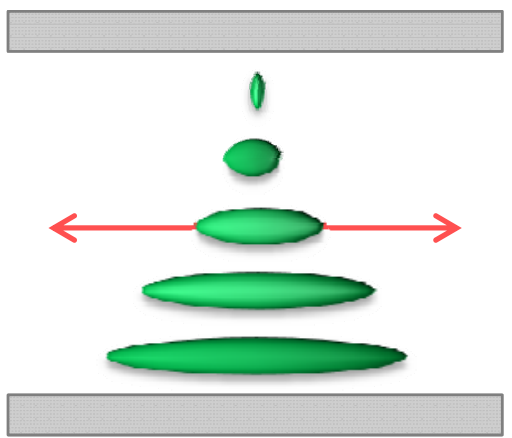


Figure 2
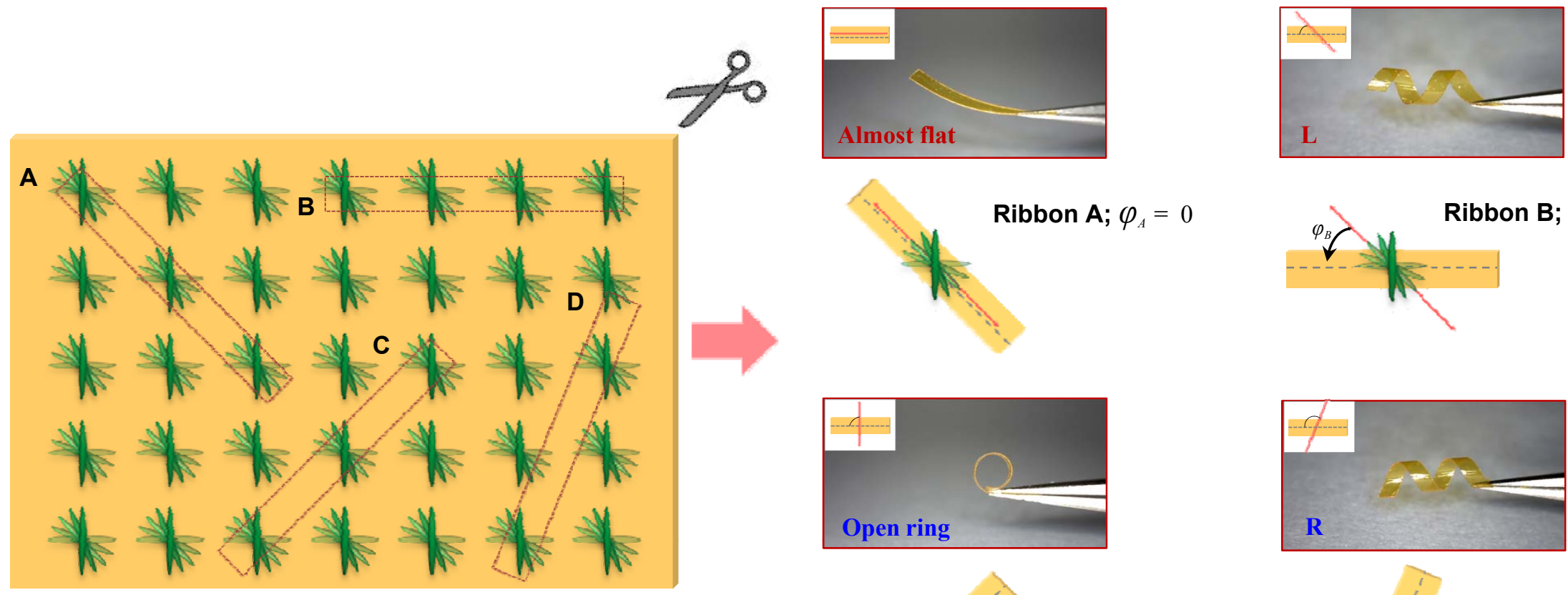

Ribbon A; $\varphi_{A}=0$

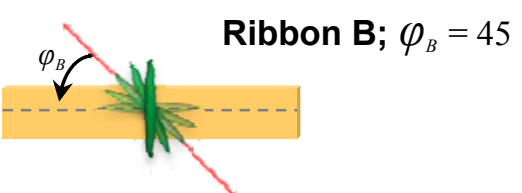

Alignment and cutting direction of the film [top view]
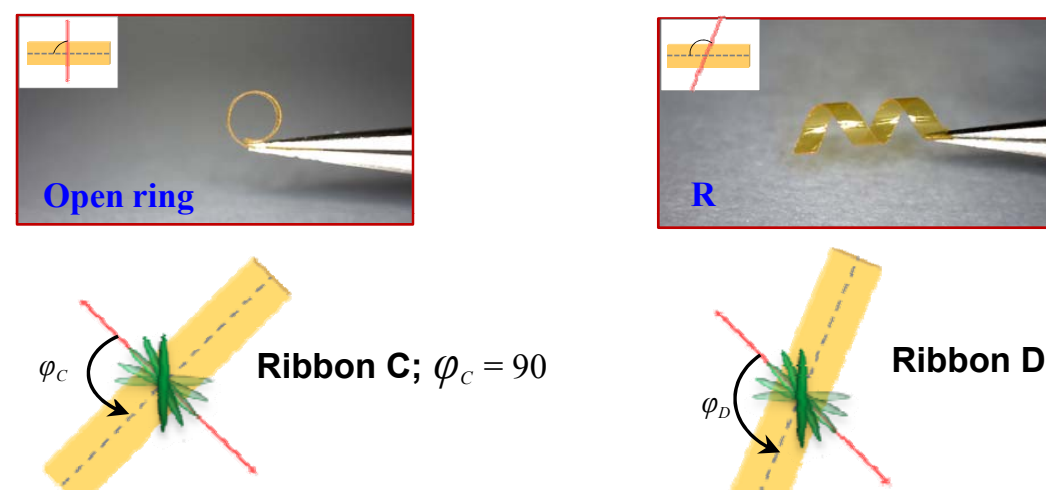

Ribbon D; $\varphi_{D}=112$ 


\section{Figure 3}

a
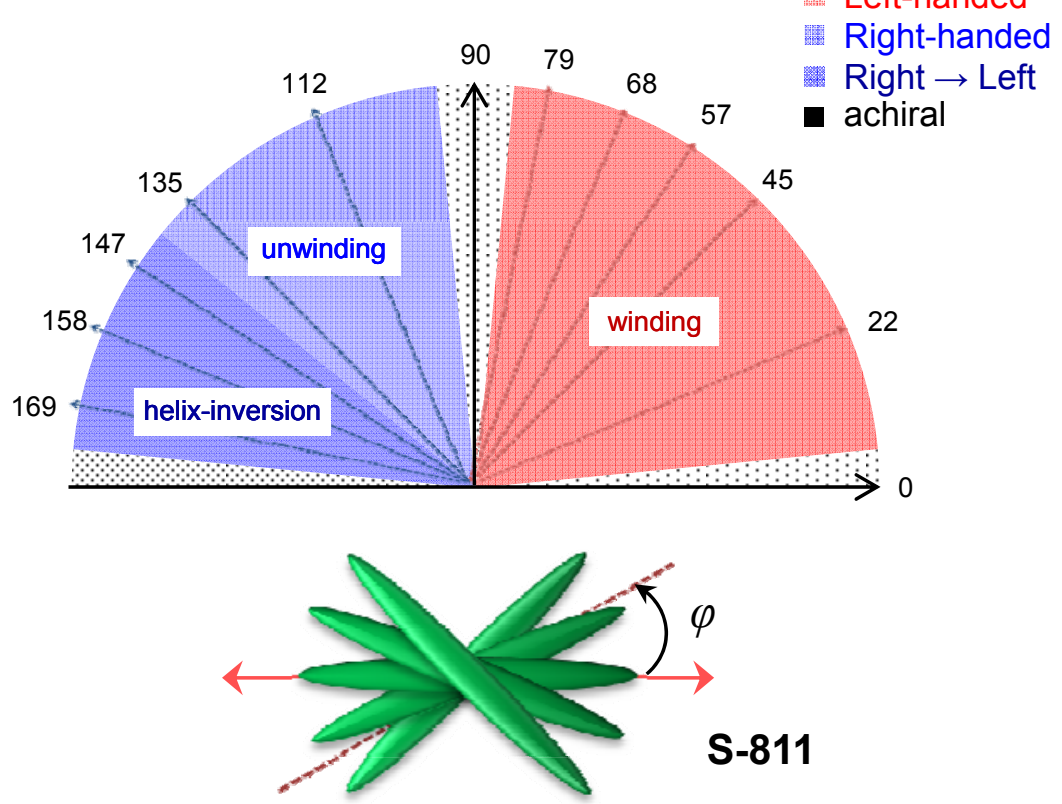

\section{b}

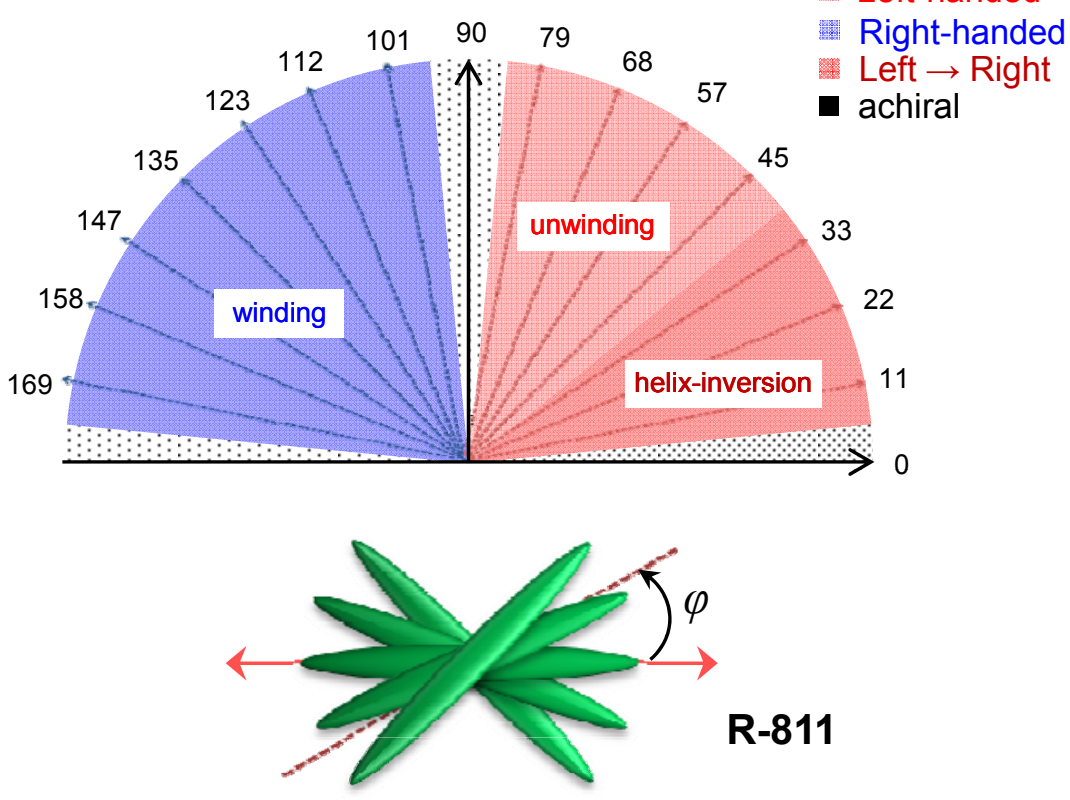


Figure 4

a
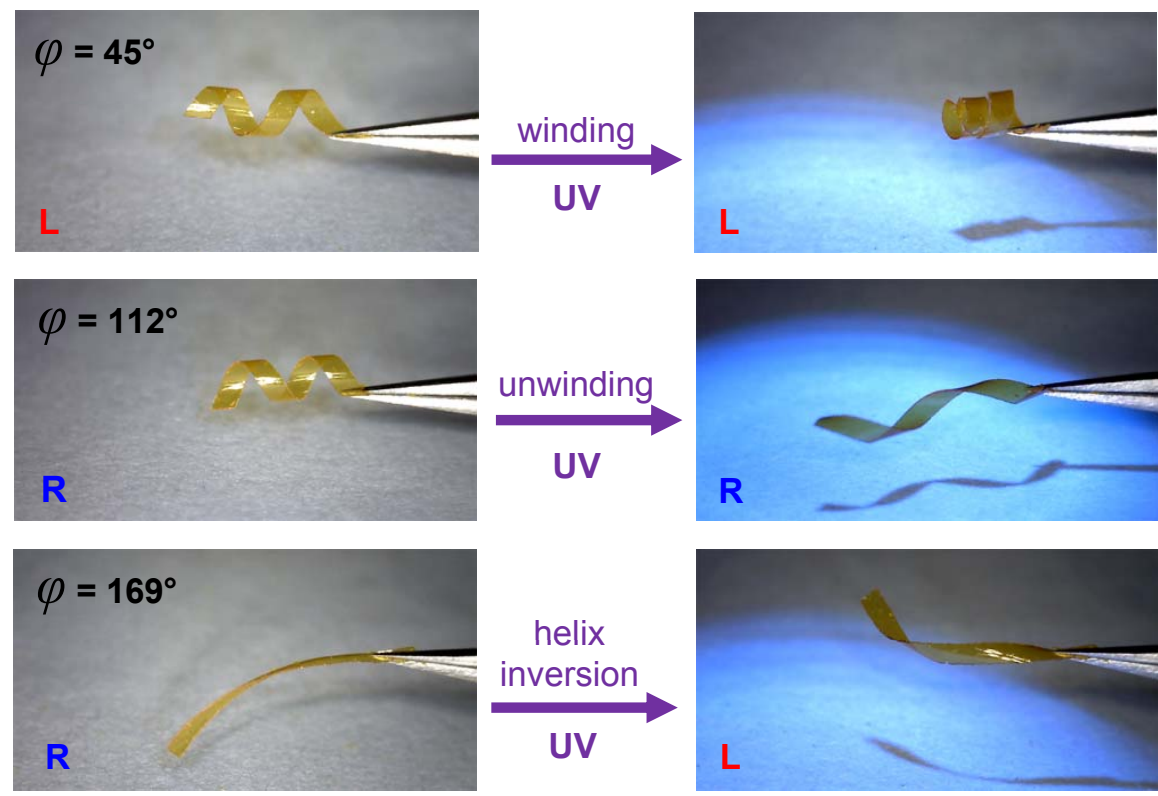

c

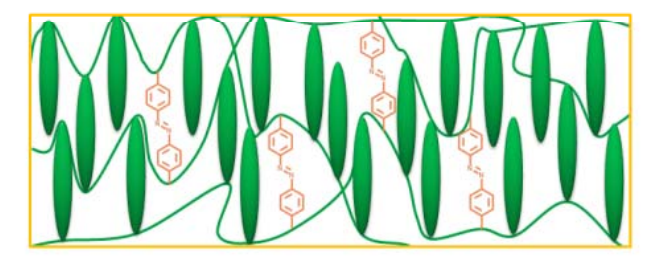

$\downarrow$ uv

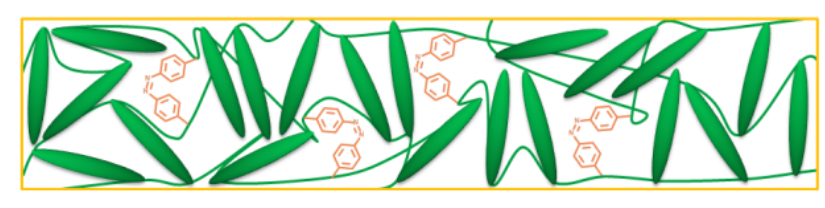

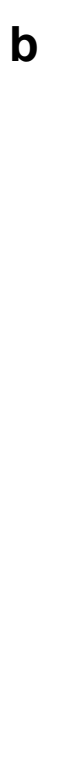

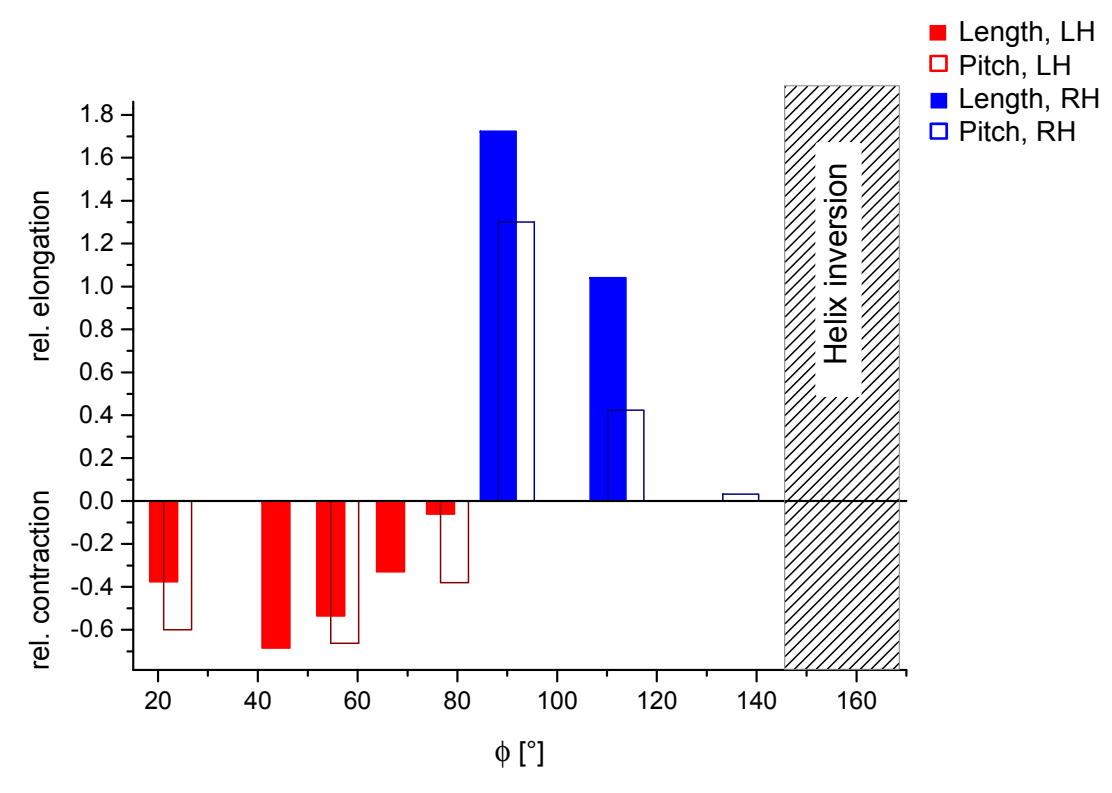

d

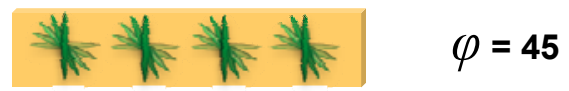

$$
\rightleftarrows\|\|
$$


Figure 5

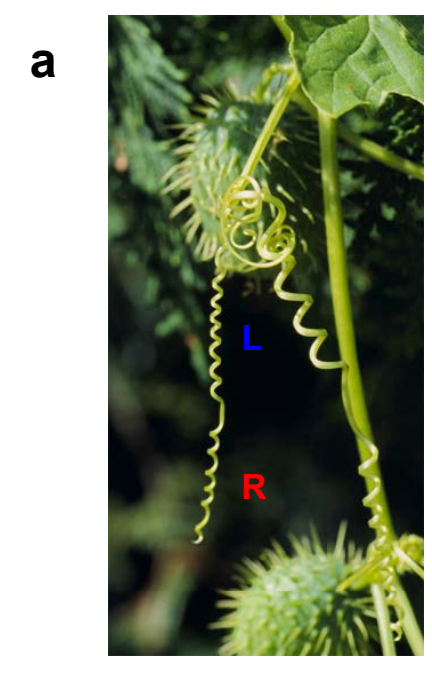

b

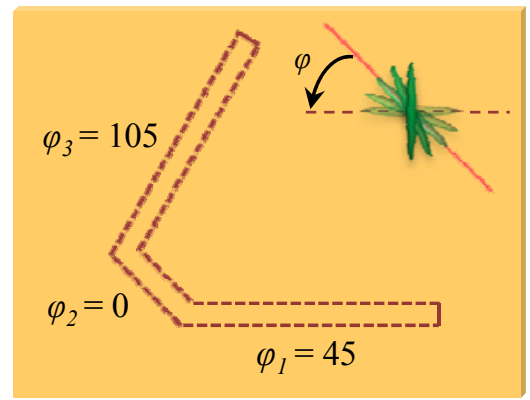

C

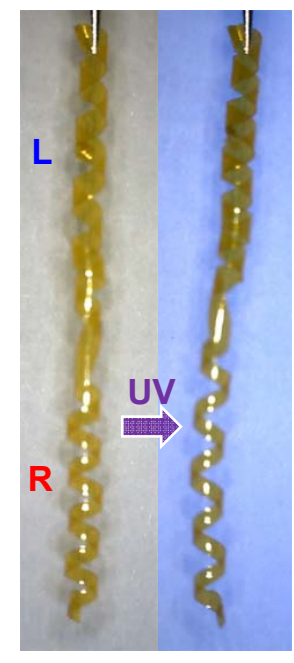

d

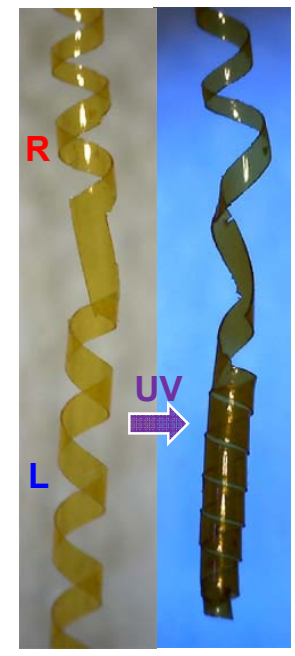

e

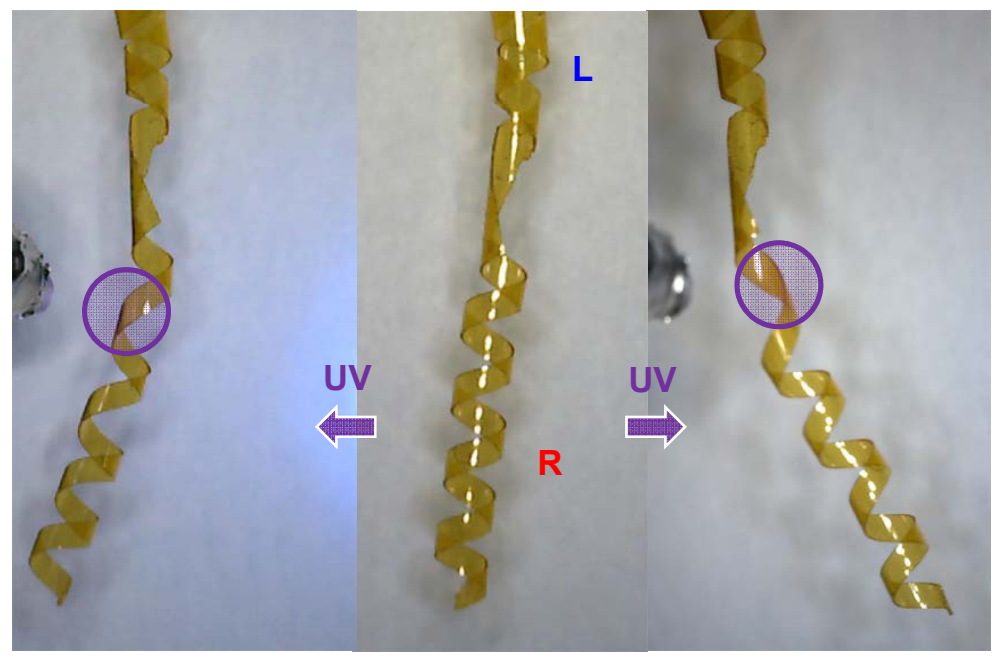


Figure 6

a

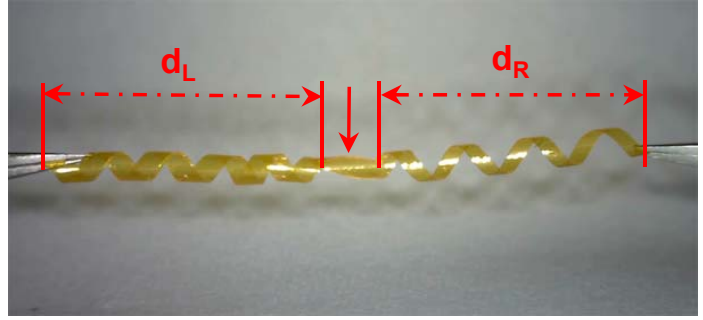

UV $(\lambda=365 \mathrm{~nm})$

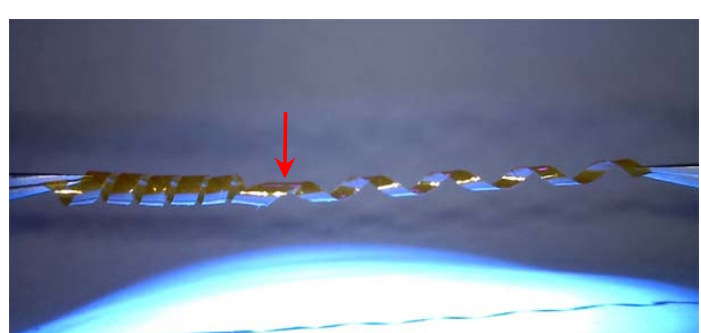

$\sqrt{ }$ visible light

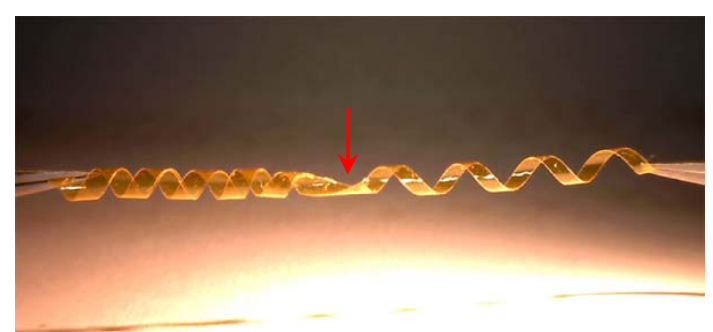

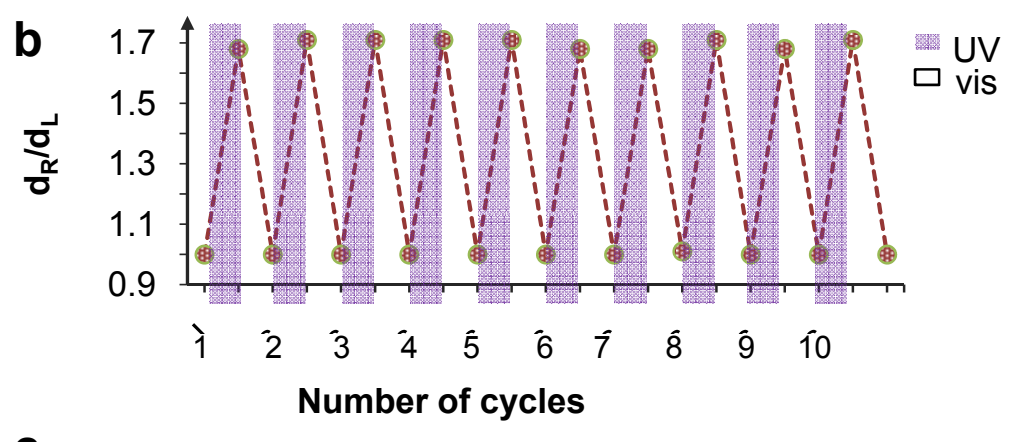

C

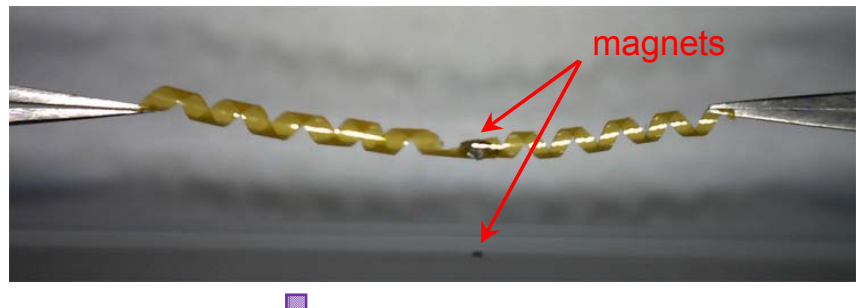

UV $(\lambda=365 \mathrm{~nm})$

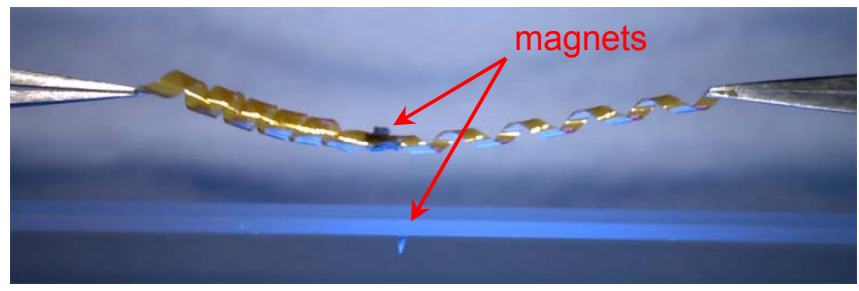




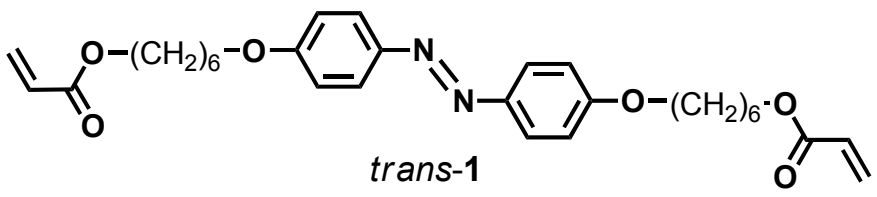

(E)-((diazene-1,2-diylbis(4,1-phenylene))bis(oxy))bis(hexane-6,1-diyl) diacrylate

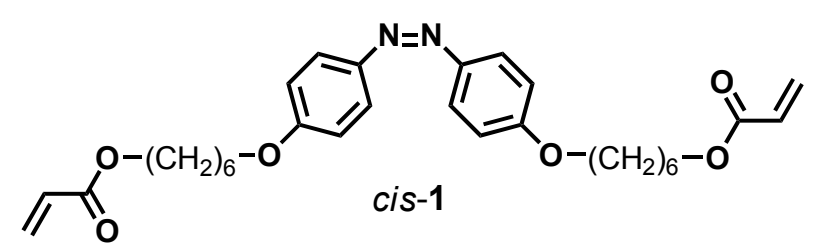

(Z)-((diazene-1,2-diylbis(4,1-phenylene))bis(oxy))bis(hexane-6,1-diyl) diacrylate

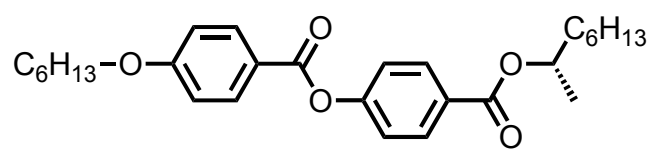

S-811

(S)-octan-2-yl 4-((4-(hexyloxy)benzoyl)oxy)benzoate

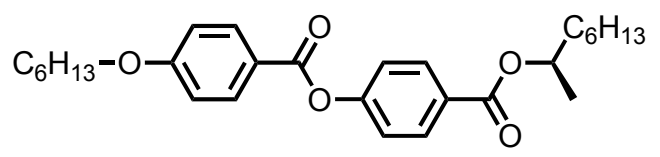

R-811

(R)-octan-2-yl 4-((4-(hexyloxy)benzoyl)oxy)benzoate 
Figure 1

a

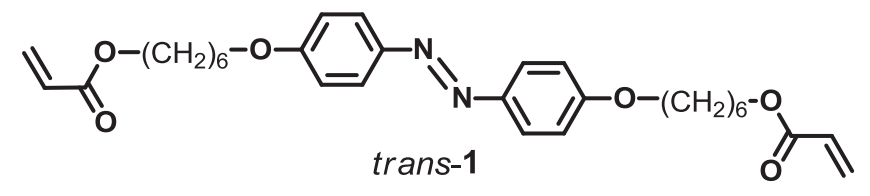

4f $\mathrm{hv}=365 \mathrm{~nm}$

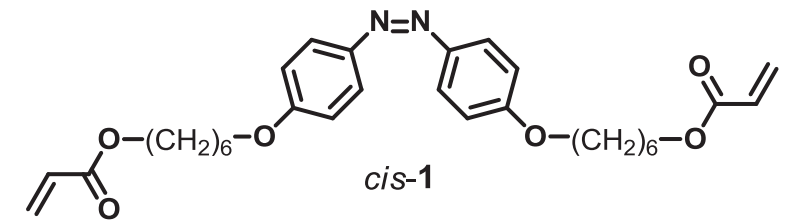

C

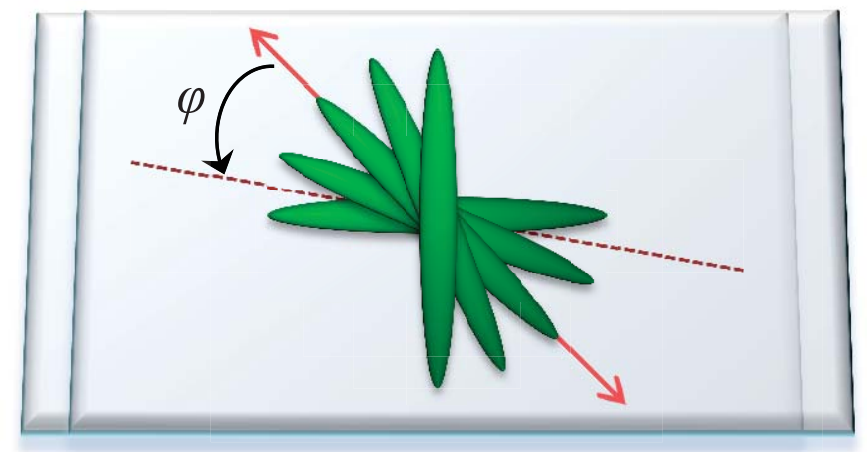

b
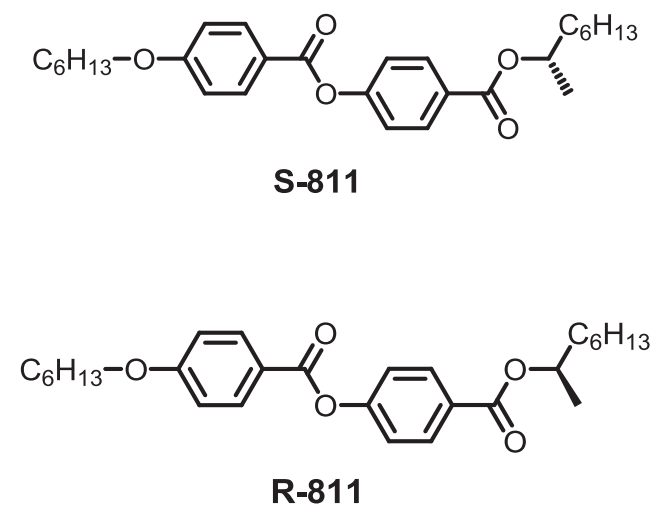

d

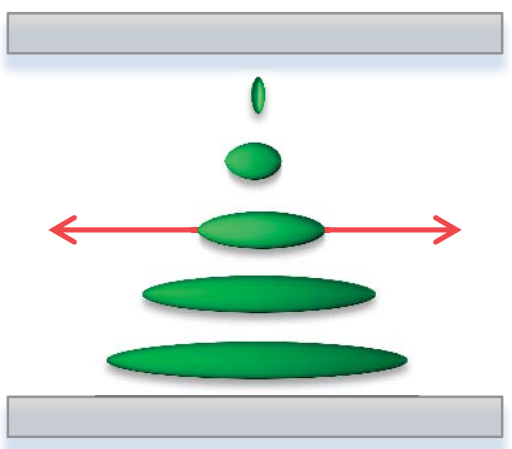


Figure 2

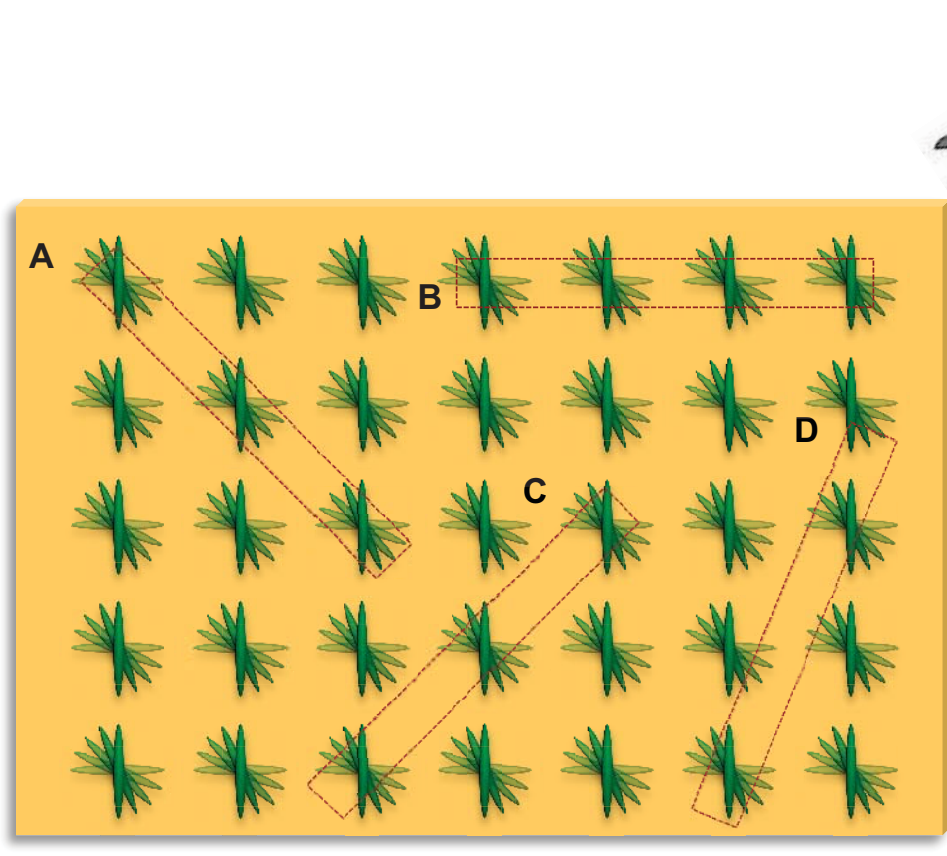

Alignment and cutting direction of the film [top view]

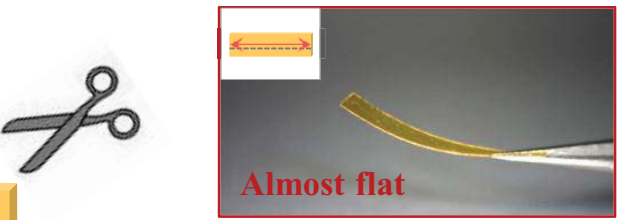

Ribbon A; $\varphi_{A}=0^{\circ}$
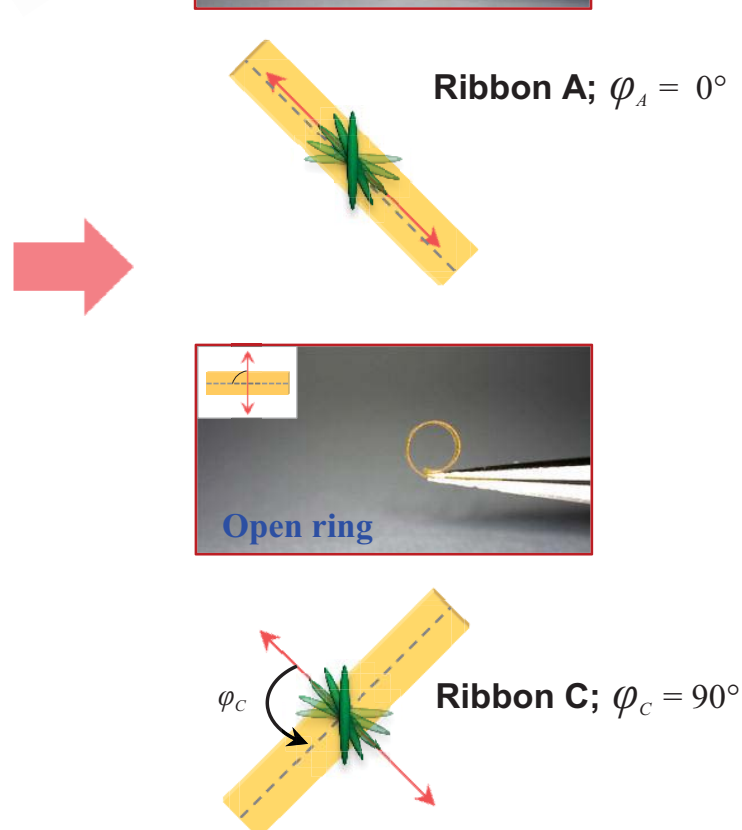
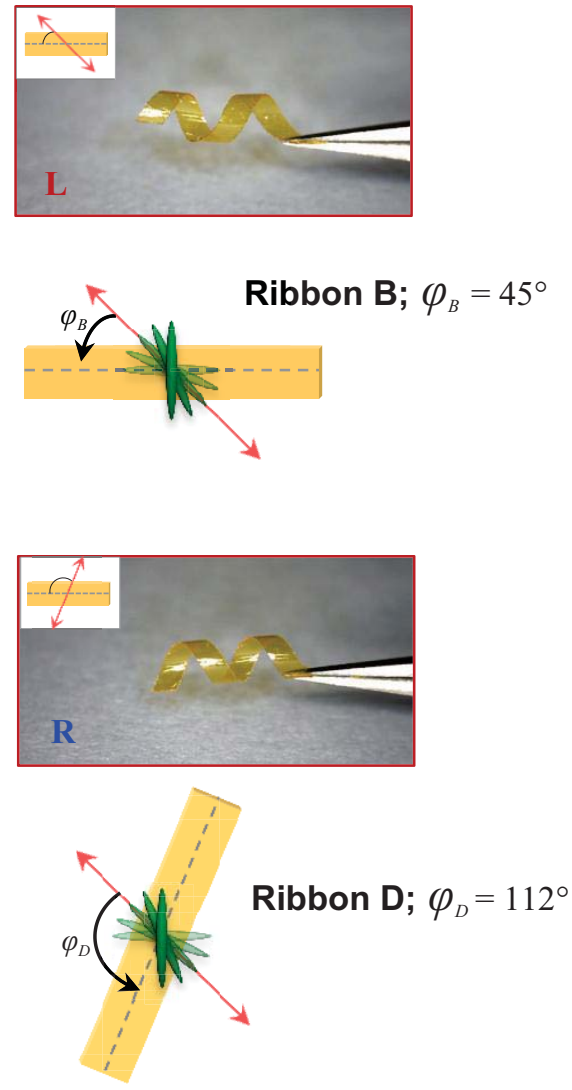


\section{Figure 3}

a
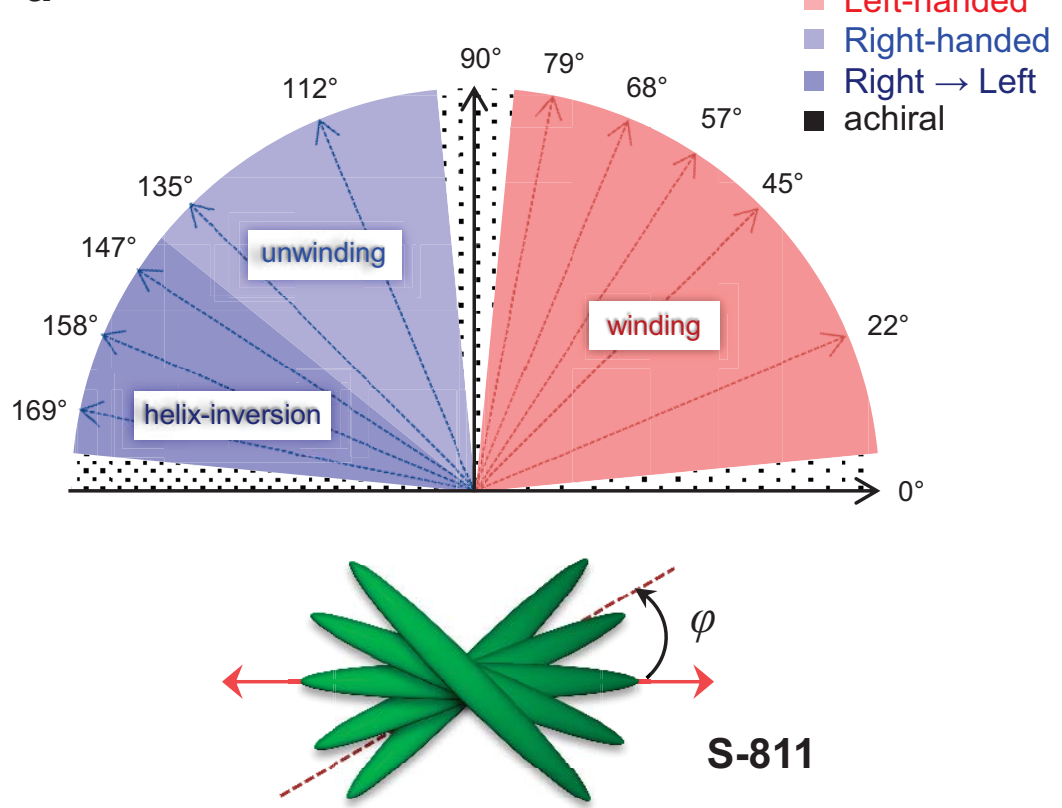

\section{b}

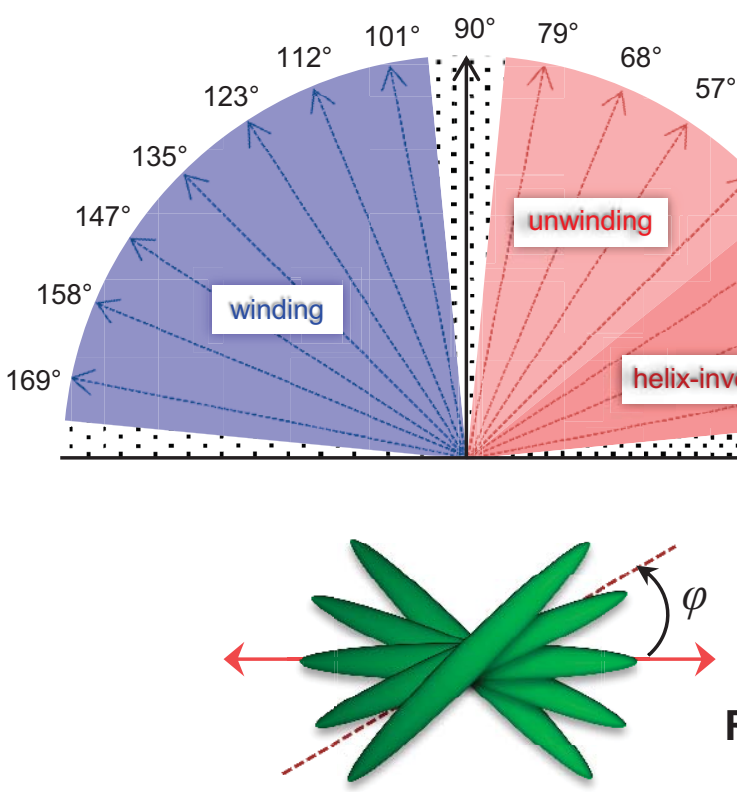

Left-handed

- Right-handed

Left $\rightarrow$ Right

- achiral $45^{\circ}$

33

$22^{\circ}$ $11^{\circ}$ 
Figure 4

a
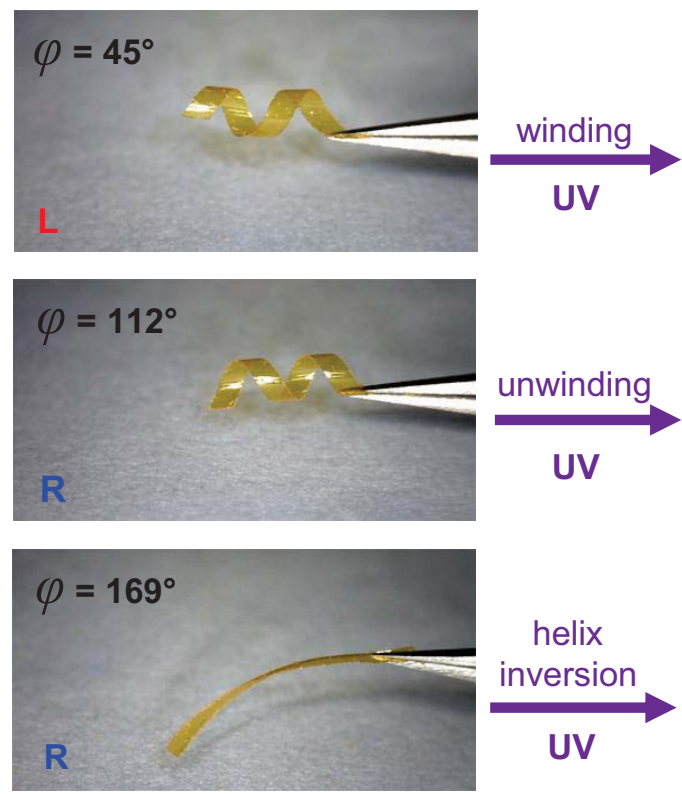

helix inversion UV

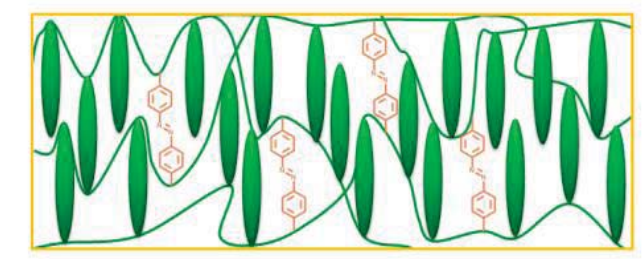

$\downarrow \mathrm{uv}$

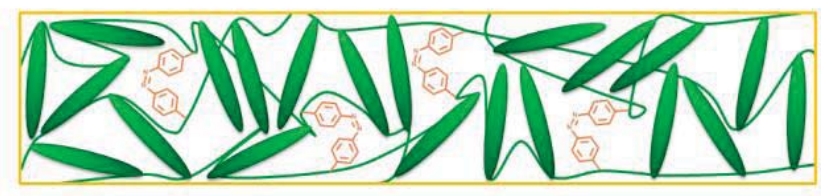

b

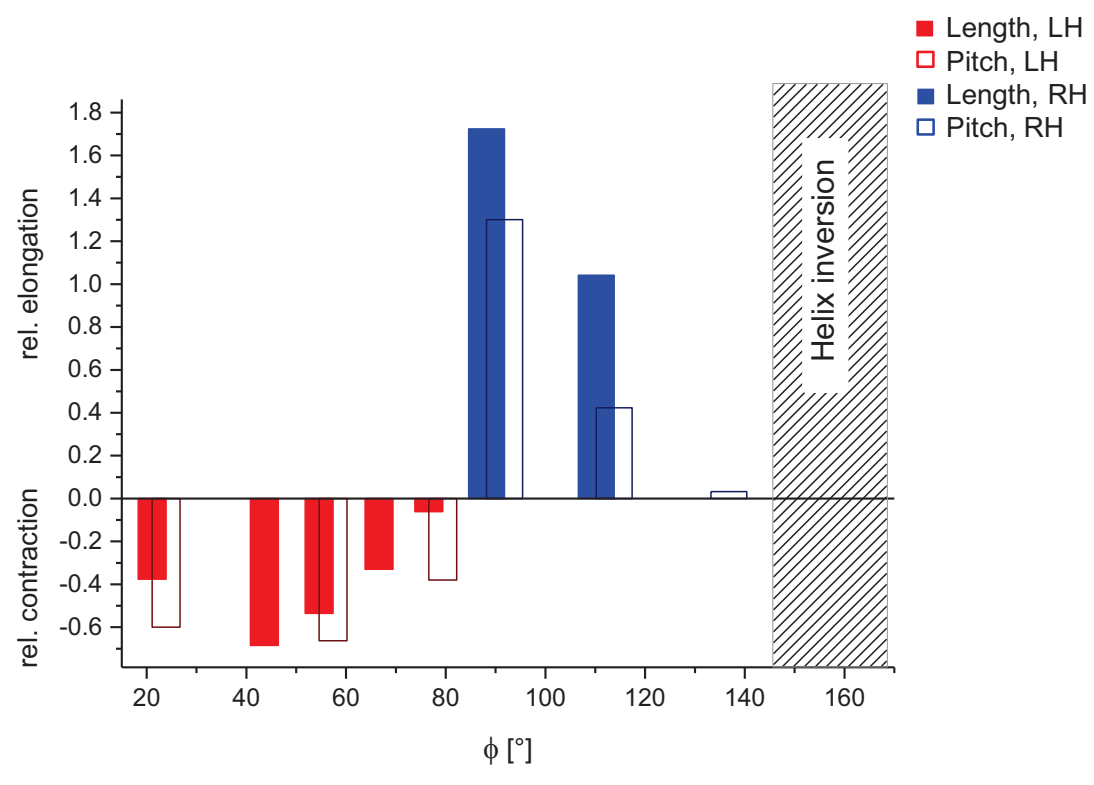

d

*ै

uv

ЕIIIIIIIIII $\Rightarrow$ Outside ofther ribon (top of the film)

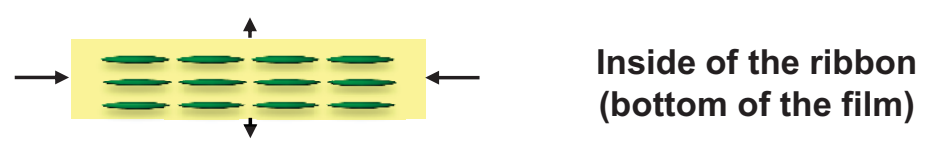


Figure 5

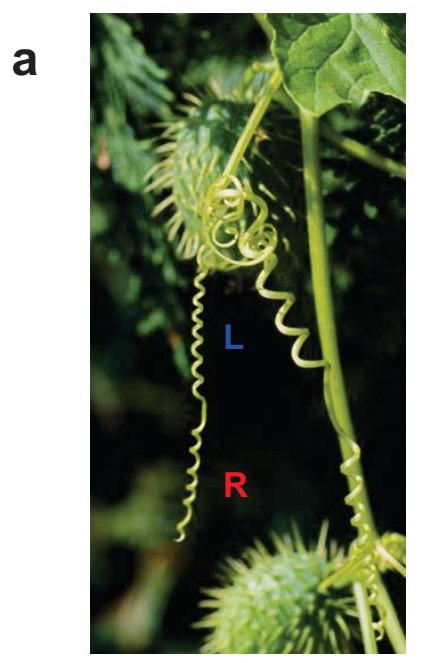

b

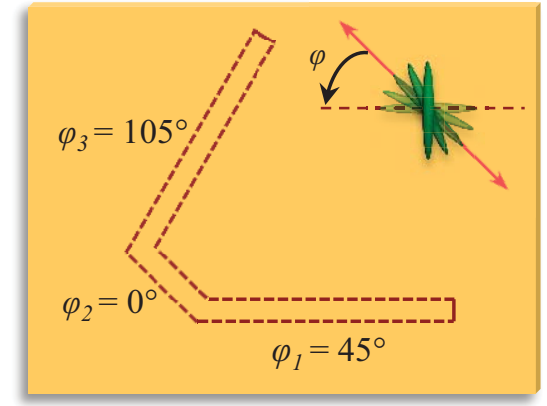

C

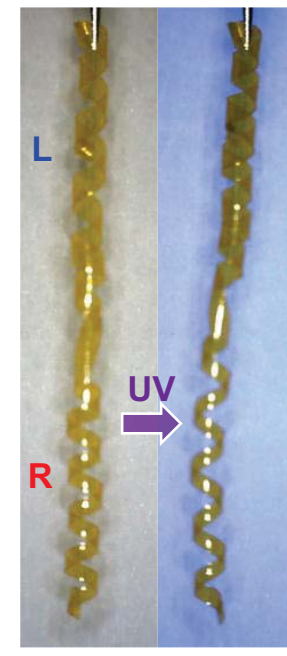

d

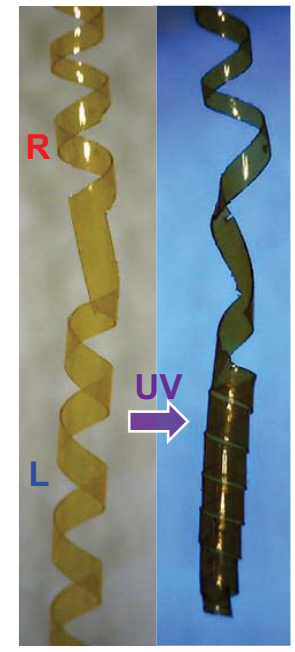

e

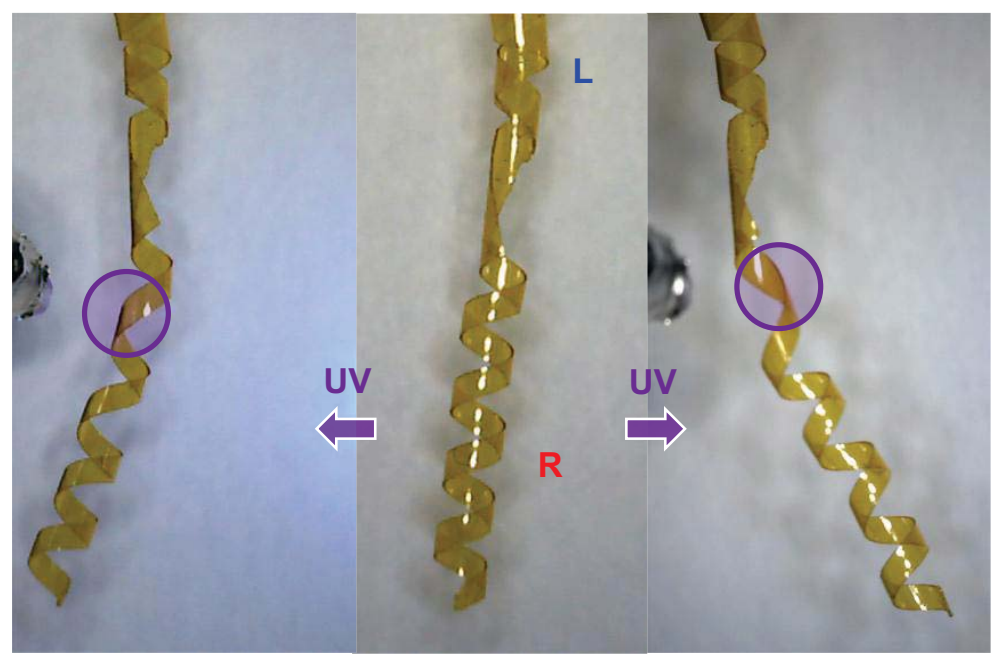


Figure 6

a

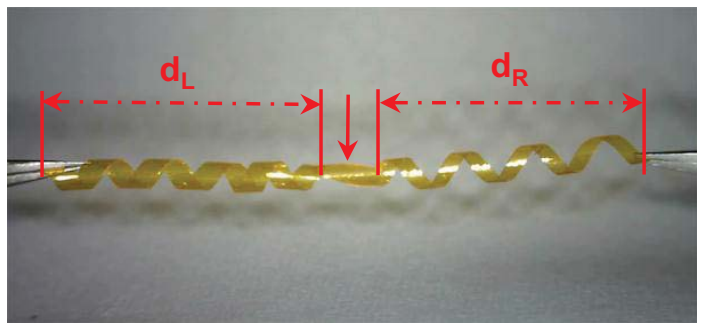

$\sqrt[U V]{ }(\lambda=365 \mathrm{~nm})$

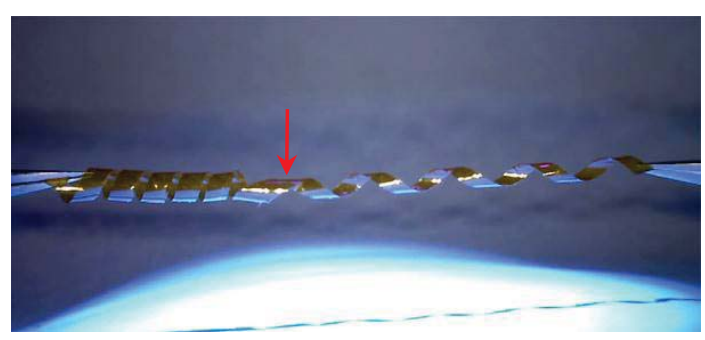

$\preceq$ visible light

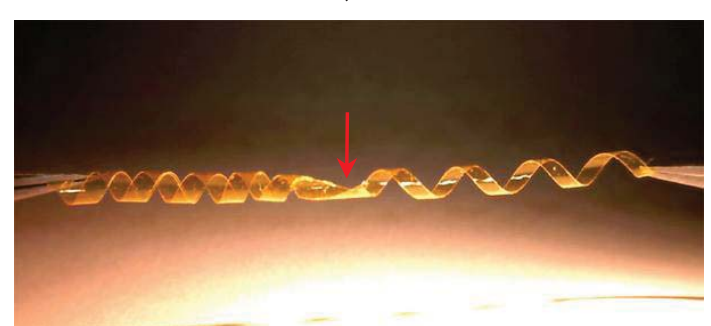

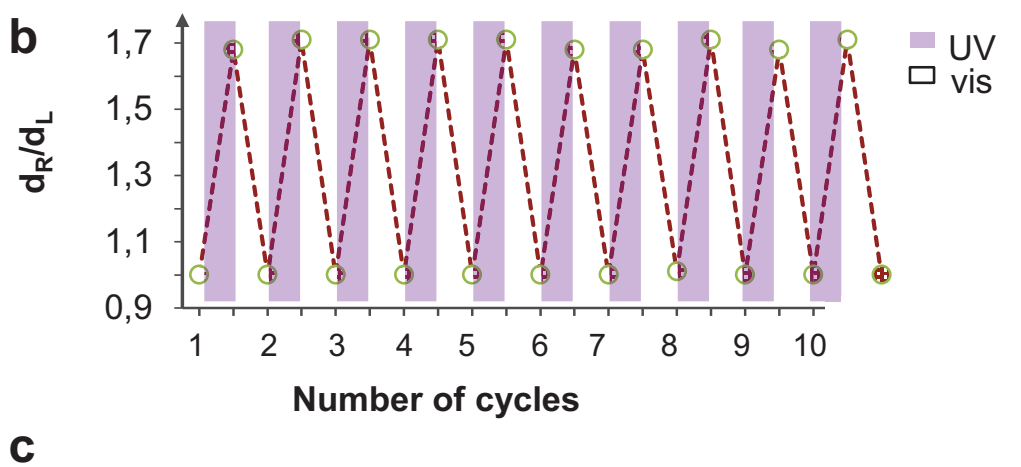

C
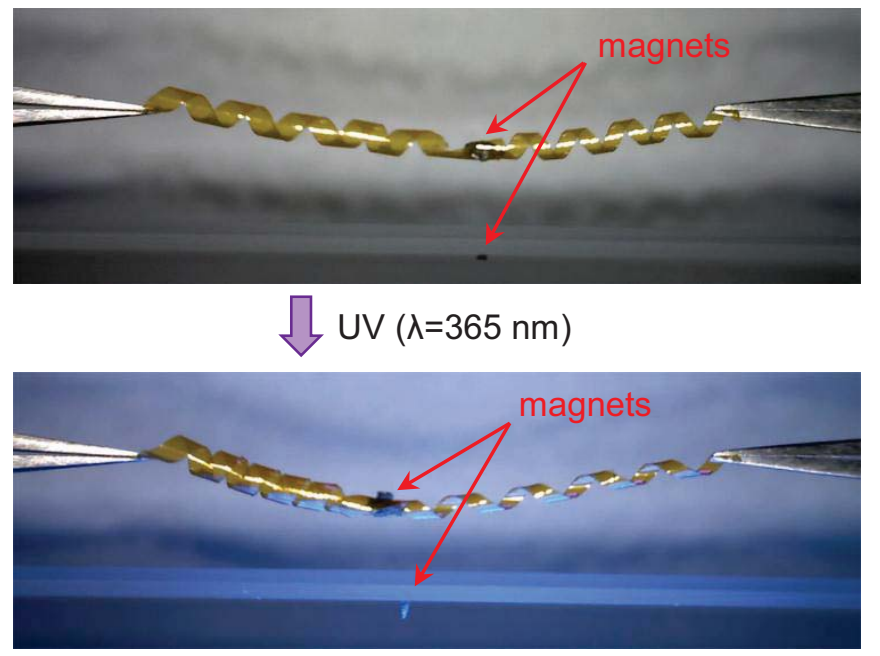
<smiles>C=C</smiles> 



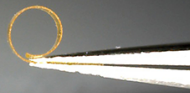




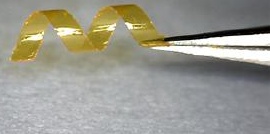




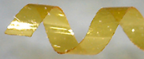
- 


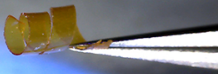




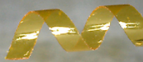

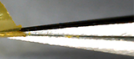

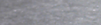

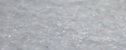




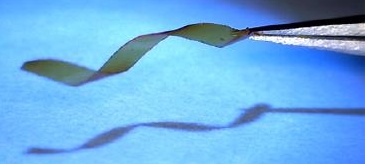



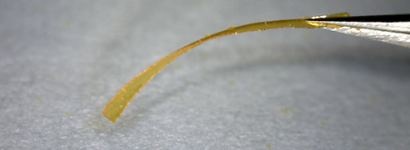

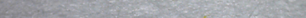


$-\infty$

-
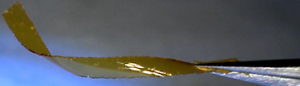

$\checkmark$

i

i

:

8

:

: 
$b$
$b$
$b$

1

1

$x^{t}$
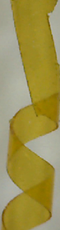
1 
$i$

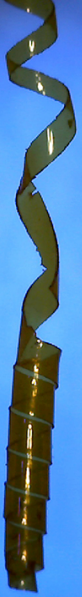




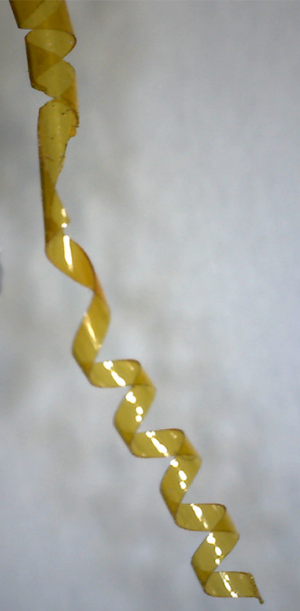


$(1)$

Y
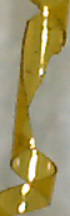

(1)

i

(a)

${ }_{0}^{6}$

, 


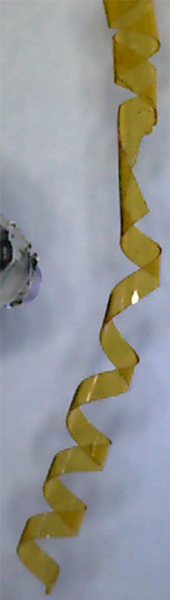




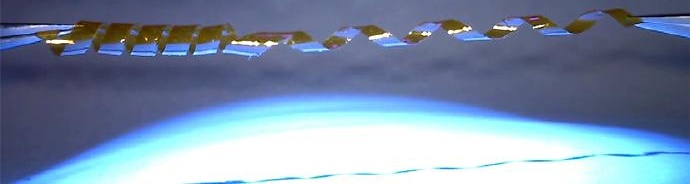




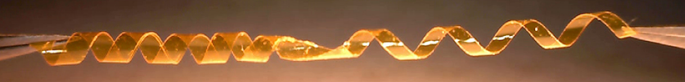



